\title{
Hypoxia inducible factor stabilizers: a promising treatment for chronic kidney disease
}

\author{
Koushiki Mani $^{1 *}$, Johnny Karini ${ }^{1}$, Kuntolika Mani $^{2}$, Ananya Amrit ${ }^{3}$
}

${ }^{1}$ Medical Review, Tata Consultancy Services, Mumbai, India

${ }^{2}$ Department of Physiology, Murshidabad Medical College and Hospital, West Bengal, India

${ }^{3}$ Department of Obstetrics \& Gynaecology, Sakra World Hospital, Bengaluru, Karnataka, India

Received: 27 August 2020

Accepted: 30 September 2020

*Correspondence:

Dr. Koushiki Mani,

Email: koushiki.cmc@gmail.com

Copyright: $\odot$ the author(s), publisher and licensee Medip Academy. This is an open-access article distributed under the terms of the Creative Commons Attribution Non-Commercial License, which permits unrestricted non-commercial use, distribution, and reproduction in any medium, provided the original work is properly cited.

\begin{abstract}
Anemia in chronic kidney disease (CKD) is a very common complication. The two main factors contributing to the development of anemia in CKD is decreased erythropoietin production and iron deficiency. Other factors that might play a role in the pathogenesis of renal anemia are: chronic inflammation leading to increased hepcidin, uremic toxins, and shorter red blood cell life span. The mainstay of treatment is iron supplementation, blood transfusion and erythropoietin stimulating agents (ESA). The discovery of hypoxia inducible factor (HIF) pathway has opened a new chapter in the treatment of anemia in CKD. The oxygen-sensitive HIF pathway plays a prominent role in the control of erythropoiesis and iron metabolism. HIF stabilizers are a new set of drugs that inhibits prolyl hydroxylase domain (PHD) proteins which are key regulators of HIF activity. Several such compounds are being developed to revolutionize the treatment of renal anemia.
\end{abstract}

Keywords: Anemia, Chronic kidney disease, Hypoxia-inducible factor, HIF stabilizers

\section{INTRODUCTION}

Anemia is a common complication of chronic kidney disease (CKD) with poor outcomes. ${ }^{1}$ The primary defect underlying anemia in CKD is relative deficiency of erythropoietin (EPO). EPO levels significantly decreases as CKD worsens. The second most important cause leading to renal anemia is iron deficiency which limits erythropoiesis. Iron deficiency in CKD can be of 2 types: functional or absolute. Functional iron deficiency is characterized by adequate iron stores but impaired iron mobilization to the bone marrow to meet the demand of excessive erythropoiesis. These patients are often marked by low serum transferrin saturation and high serum ferritin levels. Absolute iron deficiency occurs in patients with CKD due to inadequate intake of iron or poor absorption of dietary iron; chronic blood loss due to uremia-induced platelet dysfunction or uremia induced gastrointestinal hemorrhage. In addition, CKD patients on dialysis experience blood loss during routine laboratory procedures, accidental blood loss from vascular access and frequent phlebotomy. Chronic inflammatory states like CKD are often marked by increased levels of hepcidin, a small peptide produced by the liver. It plays a major role in release of iron from macrophages and intestinal iron absorption. Recent data suggests that hepcidin is one of the key regulators responsible for maintaining the iron homeostasis in our body. ${ }^{2}$

\section{HYPOXIA INDUCIBLE FACTOR PATHWAY}

HIFs are transcription factors that are responsible for cellular adaptation to hypoxia. HIF exits as a dimer, made 
up of $\alpha$-subunit and $\beta$-subunit. There are 3 HIF $\alpha$-subunit isoforms that have been identified so far namely HIF-1 $\alpha$, HIF- $2 \alpha$, and HIF- $3 \alpha .^{3-5}$ It is thought that HIF- $3 \alpha$ is a negative regulator of HIF- $1 \alpha$ and HIF- $2 \alpha .{ }^{6}$ Under normal $\mathrm{O}_{2}$ tension, HIF $\beta$-subunit is constitutively expressed but the levels of $\alpha$-subunit are low or absent. This is because prolyl hydroxylase (PHD) which acts the $\mathrm{O}_{2}$ sensor causes hydroxylation of HIF- $\alpha$ subunit. ${ }^{7}$ This hydroxylated HIF then binds to von hippel-lindau (VHL) leading to ubiquitination and proteasomal degradation. During hypoxia, PHD gets inactivated and as a result HIF- $\alpha$ subunit is no longer degraded and thus forms a stable heterodimer with HIF $\beta$-subunit. After dimerization, it translocates to the nucleus and activates gene transcription for erythropoietin.

\section{HIF and erythropoiesis}

The principal stimulus for increased RBC production in the bone marrow is erythropoietin, a glycoprotein secreted mainly from kidney and to some extent by the liver. Under hypoxic conditions, HIF binds to hypoxia response element (HRE) residing in erythropoietin gene and therefore increases erythropoiesis. Iron is an essential component for effective erythropoiesis. HIF favours iron mobilization essentially by 2 mechanisms: increasing iron uptake in the gut and inhibition of hepcidin in the liver.

In the enterocyte, HIF increases iron uptake by: 1) Increasing duodenal cytochrome b- cyt b helps in the conversion of ferric iron into ferrous iron across the brush border 2) Increasing the transcription of divalent metal transporter (DMTI)- The ferrous iron is then taken up by DMT1 across the brush border 3) Increasing ferroportin levels- under normal $\mathrm{O}_{2}$ levels, hepcidin binds to ferroportin, the only iron exporter at the basolateral membrane of the enterocyte, internalizes and degrades ferroportin. Thus, iron mobilization to plasma is limited. During hypoxia, hepcidin levels are decreased, ferroportin is now stabilized which mobilizes the iron into the circulation.

In the liver, HIF induces suppression of hepcidin therefore increasing iron availability to bone marrow.

\section{TARGETING HIF FOR TREATMENT OF ANEMIA IN CKD}

Currently, the mainstay of treatment for renal anemia is: blood transfusion, iron supplementation and erythropoietin stimulating agents (ESAs).

\section{Blood transfusion}

This is avoided to minimize the general risks associated with transfusions. In patients eligible for organ transplantation, transfusions are avoided as far as possible to reduce the risk of allosensitization.

\section{Iron supplementation}

Since renal anemia is associated with iron deficiency, iron is routinely administered in patients with CKD via oral or intravenous route. Oral administration is associated with nausea, abdominal pain, diarrhea whereas IV therapy has side effects of allergic reactions, iron overload and infections.

\section{ESAs}

Anemia management was revolutionized with the introduction of recombinant erythropoietin. Over the last decade, millions of lives of CKD were transformed with rhEPO therapy. Indeed, ESAs reduced the need for transfusions drastically. Therefore, eventually this has become the standard of care management along with iron supplementation. But it has been observed that $10-20 \%$ CKD patients fail to respond to ESAs. ${ }^{8}$ These patients have been termed as EPO resistant. Studies have also shown that ESAs are associated with cardiovascular risks, cancer-related mortality, venous thromboembolism. ${ }^{9,10}$ There is much debate regarding the 'safe' target hemoglobin levels when ESAs are administered. There is problem of hemoglobin fluctuations and often there is hemoglobin overshoot than the target level. Because of these dilemmas and controversies, ESAs are administered cautiously.

Therefore, the researchers pursued to develop a new drug that can overcome the problems with the standard therapy for renal anemia. The discovery of HIF as key regulator of erythropoiesis has led the researchers to target this pathway for anemia treatment. Novel therapeutic agents known as HIF stabilizers have been developed to reversibly inhibit prolyl hydroxylation. This creates a state of pseudo-hypoxia and thus HIF $\alpha$ is no longer degraded and is available to combine with HIF $\beta$ and upregulating erythropoiesis. Also, emerging studies have shown that HIF stabilizers inhibits hepcidin. ${ }^{11,12}$ Therefore iron absorption is increased mitigating the problem of iron deficiency classically seen in CKD patients. Preliminary data suggest that HIF stabilizers might be effective in reducing cardiovascular risks by maintaining a physiological elevation of EPO and avoiding hemoglobin overshoot brought by ESAs. Therefore, HIF stabilizers act by facilitating endogenous physiologic mechanisms. Thus, it appears promising and has triggered wide spread interest and research. There are several HIF stabilizers under development namely Roxadustat, Validustat, Daprodustat and Molidustat. The half-life of Roxadustat is 12-13 hrs with a dosing frequency of thrice weekly, half-life of Vadadustat and Daprodustat is around $4 \mathrm{hrs}$ with daily dosing and Molidustat has half-life of 10-15 hrs with daily dosing.

\section{HIF stabilizers have several advantages over current standard of treatments}


1) These are administered orally which makes it more compliant and convenient 2) These are less expensive to produce which makes it a potential candidate to reach out to a larger mass 3) These are stable at room temperature which removes the need for cold-chain transport and 4) It also reduces the need for iron supplementation because it improves the iron deficient state of CKD by modifying the effect of hepcidin.

\section{Safety concerns of HIF stabilizers}

Apart from erythropoiesis, HIFs regulate numerous cellular and biological processes namely, angiogenesis, glucose, fatty acid, cholesterol and mitochondrial metabolism, signaling pathways that control cell growth and cell death, cardiovascular functions, inflammation, cell motility and matrix production. Therefore, although HIF stabilizers have been well tolerated and showed promising data in short-term clinical trials, the nonerythropoietic effects of HIF raise some safety concerns. These pleotropic activities of HIF have the potential to precipitate some serious adverse effect that can impact the quality of life of CKD patients.

\section{Role of HIF in pulmonary hypertension}

In response to hypoxia, the systemic circulation undergoes vasodilation to meet the increased $\mathrm{O} 2$ demands of the tissues. On the contrary, the pulmonary vessels constrict when the $\mathrm{O} 2$ tension decreases, also known as hypoxic pulmonary vasoconstriction (HPV). It is observed that in the setting of chronic hypoxia, there is pulmonary vasculature remodelling leading to pulmonary hypertension. Now, the fact that pulmonary vasculature contracts immediately under hypoxia support the idea that this is more of an acute response rather than a transcription level change. But preliminary evidences suggest that HIF might play role in the pathogenesis of HPV. Loss-of-function mutation in HIF leads to reduced pulmonary arterial pressure whereas, genetic mutation causing HIF overexpression was found to correlate with pulmonary hypertension. Also, individuals with Chuvash polycythaemia, where VHL is deleted, presented with high HIF levels and subsequent development of PH. Thus, these findings suggest that we need more data to study the implications of HIF stabilizers on pulmonary vasculature.

\section{Role of HIF in angiogenesis and cancer}

Emerging evidence suggest that HIF plays a role in the pathogenesis of cancer. HIF $1-\alpha$ overexpression occurs in cancers of breast, bladder, cervix, colon, endometrium, and stomach. Hypoxia, a hallmark of the tumor mileu is responsible for the aggressiveness of the tumor. Tumor cells adapt to this low $\mathrm{O} 2$ tension by activating the HIF pathway. HIF-1 $\alpha$ levels are greatly increased in rapidly dividing and poorly vascularized tumors. In the tumor microenvironment, activation of HIF induces the transcription of several pro-angiogenetic factors such as
VEGF, a major driver of tumor angiogenesis. ${ }^{16} \mathrm{HIFs}$ also play a role in the regulation of tumor cell growth and metastasis. ${ }^{17}$ Activation of HIF signaling in malignant cells has been associated with tumor initiation and progression. Also, there are concerns, that HIF stabilizers might exacerbate retinal angiogenesis in diabetic retinopathy associated with CKD.

\section{CONCLUSION}

Thus, to conclude, HIF stabilizers have shown to be quite effective in treating anemia associated with CKD and decreasing the progression of renal disease. But there is paucity of robust data in regard to the pleotropic effects of HIF. The association between HIF stabilizers and upregulation of various signalling pathways is theoretical, but it has the potential to set off various unwanted offtarget effects. Therefore, studies are required to understand the long-term effects of HIF stabilizers.

Funding: No funding sources Conflict of interest: None declared Ethical approval: Not required

\section{REFERENCES}

1. Babitt JL, Lin HY. Mechanisms of Anemia in CKD. J Am Soc Nephrol. 2012; 23:28:3458456.

2. Babitt JL, Lin HY. Molecular mechanisms of hepcidin regulation: Implications for the anemia of CKD. Am J Kidney Dis. 2010;55:726-41.

3. Semenza GL. Regulation of mammalian O2 homeostasis by hypoxia-inducible factor 1 . Annu Rev Cell Dev Biol. 1999;15:551-78.

4. Kewley RJ, Whitelaw ML, Chapman-Smith A. The mammalian basic helix-loop-helix/PAS family of transcriptional regulators. Int $\mathrm{J}$ Biochem Cell Biol. 2004; 36:189-204.

5. Wenger RH, Stiehl DP, Camenisch G. Integration of oxygen signaling at the consensus HRE. Scien Sign. 2005;2005:12.

6. Hara S, Hamada J, Kobayashi C, Kondo Y and Imura $\mathrm{N}$ : Expression and characterization of hypoxiainducible factor (HIF)-3alpha in human kidney: Suppression of HIF-mediated gene expression by HIF-3alpha. Biochem Biophys Res Commun. 2001;287:808-13.

7. Kaelin WG Jr, Ratcliffe PJ. Oxygen sensing by metazoans: the central role of the HIF hydroxylase pathway. Mol Cell. 2008;30:393-402.

8. Eschbach JW, Egrie JC, Downing MR, Browne JK, Adamson JW. Correction of the anemia of end-stage renal disease with recombinant human erythropoietin. Results of a combined phase I and II clinical trial. N Engl J Med. 1987;316:73-8.

9. Singh AK, Szczech L, Tang KL, Barnhart H, Sapp S, Wolfson M, Reddan D; CHOIR Investigators: Correction of anemia with epoetin alfa in chronic kidney disease. N Engl J Med. 2006;355:2085-98. 
10. Solomon SD, Uno H, Lewis EF, Eckardt KU, Lin J, Burdmann EA, et al. Trial to Reduce Cardiovascular Events with Aranesp Therapy (TREAT) Investigators: Erythropoietic response and outcomes in kidney disease and type 2 diabetes. N Engl J Med. 2010;363:1146-55.

11. Liu Q, Davidoff O, Niss K, Haase VH. Hypoxiainducible factor regulates hepcidin via erythropoietininduced erythropoiesis. J Clin Invest. 2012; 122:4635-44.

12. Kautz L, Jung G, Valore EV, Rivella S, Nemeth E, Ganz T. Identification of erythroferrone as an erythroid regulator of iron metabolism. Nat Genet. 2014;46:678-84.

13. Dai Z, Li M, Wharton J, Zhu MM, Zhao YY. Prolyl4 hydroxylase 2 (PHD2) deficiency in endothelial cells and hematopoietic cells induces obliterative vascular remodeling and severe pulmonary arterial hypertension in mice and humans through hypoxiainducible factor-2 $\alpha$. Circulat. 2016;133:2447-58.
14. Tang H, Babicheva A, McDermott KM, Gu Y, Ayon RJ, Song S, et al. Endothelial HIF-2 $\alpha$ contributes to severe pulmonary hypertension due to endothelial-tomesenchymal transition. Americ J Physiol Lun Cellul Molecul Physiol. 2018;314(2):L256-75.

15. Liu S, Kumar SM, Martin JS, Yang R, Xu X. Snail1 mediates hypoxia-induced melanoma progression. Am J Pathol. 2011;179:3020-31.

16. Nagaraju GP, Bramhachari PV, Raghu G, El-Rayes BF. Hypoxia inducible factor-1alpha: Its role in colorectal carcinogenesis and metastasis. Canc lette. 2015;366(1):11-8.

17. Maxwell PH, Pugh CW, Ratcliffe PJ. Activation of the HIF pathway in cancer. Curr Opin Genet Dev. 2001;11(3):293-9.

Cite this article as: Mani $\mathrm{K}$, Karini J, Mani $\mathrm{K}$, Amrit A. Hypoxia inducible factor stabilizers: a promising treatment for chronic kidney disease. Int J Basic Clin Pharmacol 2020;9:1766-9. 